International Journal of Theoretical and Applied Finance

Vol. 18, No. 8 (2015) 1599001 (4 pages)

(C) World Scientific Publishing Company

DOI: $10.1142 / \mathrm{S} 0219024915990010$

\title{
Author Index Volume 18 (2015)
}

Al-Fagih, L., The British Knock-Out Put Option Alòs, E., de Santiago, R. \& Vives, J., Calibration of Stochastic Volatility Models via SecondOrder Approximation: The Heston Case

Anderluh, J. H. M., see Shen, Y.

Arunachalam, V., see Jiménez, J. A.

Assonken, P. \& Ladde, G. S., Option Pricing with a Levy-Type Stochastic Dynamic Model for Stock Price Process Under Semi-Markovian Structural Perturbations

Auer, B. R., On the Role of Skewness, Kurtosis, and the Location and Scale Condition in a Sharpe Ratio Performance Evaluation Setting

Barucci, E. \& Marazzina, D., Risk Seeking, Nonconvex Remuneration and Regime Switching

Benth, F. E. \& Blanco, S. A. S., Forward Prices as Functionals of the Spot Path in Commodity Markets Modeled by Levy Semistationary Processes

Benth, F. E. \& OrtizLatorre, S., A Change of Measure Preserving the Affine Structure in the Barndorff-Nielsen and Shephard Model for Commodity Markets

Bernis, G., Carassus, L., Docq, G. \& Scotti, S.,

\author{
2 (2015) 1550008 \\ 6 (2015) 1550036 \\ 1 (2015) 1550001 \\ 8 (2015) 1550051
}

8 (2015) 1550052

6 (2015) 1550037

2 (2015) 1550009

2 (2015) 1550010

6 (2015) 1550038
Optimal Credit Allocation Under Regime Uncertainty with Sensitivity Analysis

Biagini, F., Bregman, J. \& Meyer-Brandis, T., Electricity Futures Price Modeling with Lévy Term Structure Models

Blanchet-Scalliet, C., Chevalier, E., Kharroubi, I. \& Lim, T., Max-Min Optimization Problem for Variable Annuities Pricing

Blanco, S. A. S., see Benth, F. E.

Bouchaud, J.-P., see Vargas, $\mathrm{V}$.

Bregman, J., see Biagini, F.

Brigo, D., Buescu, C., Pallavicini, A. \& Liu, Q., A Note on the SelfFinancing Condition for Funding, Collateral and Discounting

Brigo, D., Garcia, J. \& Pede, N., CoCo Bonds Pricing with Credit and Equity Calibrated First-Passage Firm Value Models

Brody, D. C. \& Hadjipetri, S., Coherent Chaos Interest-Rate Models

Buescu, C., see Brigo, D.

Carassus, L., see Bernis, G.

Chan, L., see Elliott, R. J.

Cheang, G. H. L., see Mina, K. F.

Cherif, S. M. L. B., see Eddahbi, M.
1 (2015) 1550002

1 (2015) 1550003

8 (2015) 1550053

$2(2015) 1550010$

4 (2015) 1550022

$1(2015) 1550003$

2 (2015) 1550011

3 (2015) 1550015

3 (2015) 1550016

2 (2015) 1550011

1 (2015) 1550002

4 (2015) 1550023

4 (2015) 1550024

6 (2015) 1550039 
Chetalova, D., Schmitt, T. A., Schäfer, R. \& Guhr, T., Portfolio Return Distributions: Sample Statistics with Stochastic Correlations

Chevalier, E., see Blanchet-Scalliet, C.

Chiarella, C., see Mina, K. F.

Cordero, F. \& PerezOstafe, L., Critical Transaction Costs and 1-Step Asymptotic Arbitrage in Fractional Binary Markets

Curme, C., Stanley, H. E. \& Vodenska, I., Coupled Network Approach to Predictability of Financial Market Returns and News Sentiments

Dao, T.-L., see Vargas, V.

de Santiago, R., see Alòs, E.

Desmettre, S., Korn, R. \& Seifried, F. T., Lifetime Consumption and Investment for WorstCase Crash Scenarios

Dette, H., see Schmitt, T. A.

Docq, G., see Bernis, G.

Eddahbi, M., Cherif, S. M. L. B. \& Nasroallah, A., Computation of Greeks for JumpDiffusion Models

Ekström, E. \& Lu, B., Short-Time Implied Volatility in Exponential Lévy Models

El Hajjaji, O. \& Subbotin, A., CVA with Wrong Way Risk: Sensitivities, Volatility and Hedging

Elliott, R. J., Chan, L. \& Siu, T. K., A Dupire Equation for a RegimeSwitching Model

Escobar, M., Götz, B. Neykova, D. \& Zagst, R., Pricing Two-Asset Barrier Options Under Stochastic Correlation via Perturbation

Escobar, M., Neykova, D. \& Zagst, R., Portfolio Optimization in Affine Models with Markov Switching
2 (2015) 1550012

8 (2015) 1550053

4 (2015) 1550024

5 (2015) 1550029

7 (2015) 1550043

4 (2015) 1550022

6 (2015) 1550036

1 (2015) 1550004

7 (2015) 1550044

1 (2015) 1550002

6 (2015) 1550039

4 (2015) 1550025

3 (2015) 1550017

4 (2015) 1550023

3 (2015) 1550018

5 (2015) 1550030
Feng, R. \& Volkmer, H. W., Conditional Asian Options

Fontana, C., Weak and Strong No-Arbitrage Conditions for Continuous Financial Markets

Funahashi, H., An Analytical Approximation for European Option Prices under Stochastic Interest Rates

Garcia, J., see Brigo, D.

Goard, J., see Oud, M. A. A.

Götz, B., see Escobar, M.

Gremm, M., The StressDependent Random Walk

Grzelak, L. A., see Van der Stoep, A. W.

Guéant, O., Pu, J. \& Royer, G., Accelerated Share Repurchase: Pricing and Execution Strategy

Guhr, T., see Chetalova, D.

Guhr, T., see Schmitt, T. A.

Gulisashvili, A., LeftWing Asymptotics of the Implied Volatility in the Presence of Atoms

Haba, F. \& Jacquier, A., Asymptotic Arbitrage in the Heston Model

Hadjipetri, S., see Brody, D. C.

Hambly, B. \& Vaicenavicius, J., The $3 / 2$ Model as a Stochastic Volatility Approximation for a Large-Basket PriceWeighted Index

Hinnerich, M., Consistent Parallel and Proportional Shifts in the Term Structure of Futures Prices

Horsky, R. \& Sayer, T., Joining the Heston and a Three-Factor Short Rate Model: A ClosedForm Approach

Itkin, A., High Order Splitting Methods for Forward PDEs and PIDEs

Jacquier, A., see Haba, F.
6 (2015) 1550040

1 (2015) 1550005

4 (2015) 1550026

3 (2015) 1550015

8 (2015) 1550050

3 (2015) 1550018

8 (2015) 1550054

6 (2015) 1550042

3 (2015) 1550019

2 (2015) 1550012

7 (2015) 1550044

2 (2015) 1550013

8 (2015) 1550055

3 (2015) 1550016

6 (2015) 1550041

1 (2015) 1550006

8 (2015) 1550056

5 (2015) 1550031

8 (2015) 1550055 
Jeanblanc, M. \& Leniec, M., Role of Information in Pricing DefaultSensitive Contingent Claims

Jeanblanc, M., Mastrolia, T., Possamaï, D \& Réveillac, A., Utility Maximization with Random Horizon: A BSDE Approach

Jiménez, J. A., Arunachalam, V. \& Serna, G. M., Option Pricing Based on a Log-Skew-Normal Mixture

Kchia, Y. \& Protter, P., Progressive Filtration Expansions via a Process, with Applications to Insider Trading

Kharroubi, I., see Blanchet-Scalliet, C.

Korn, R., see Desmettre, S.

Kwok, Y. K., see Zeng, P.

Ladde, G. S., see Assonken, P.

Leniec, M., see Jeanblanc, M.

Leung, T. \& Li, X., Optimal Mean Reversion Trading with Transaction Costs and StopLoss Exit

Leung, T., Yamazaki, K. \& Zhang, H., An Analytic Recursive Method for Optimal Multiple Stopping: Canadization and Phase-Type Fitting

Li, X., see Leung, T.

Lim, T., see BlanchetScalliet, C.

Liu, Q., see Brigo, D.

Lu, B., see Ekström, E.

Marazzina, D., see Barucci, E.

Mastrolia, T., see Jeanblanc, $\mathrm{M}$.

Melnikov, A. \& Nosrati, A., Efficient Hedging for Defaultable Securities and Its Application to Equity-Linked Life Insurance Contracts

Menoukeu-Pamen, O. \& Momeya, R., Local Risk-Minimization Under Markov-Modulated
1 (2015) 1550007 Mina, K. F., Cheang, G. H. L. \& Chiarella, C., Approximate Hedging of Options Under Jump-Diffusion Processes

7 (2015) 1550045

Momeya, R., see Menoukeu-Pamen, $\mathrm{O}$.

Nasroallah, A., see Eddahbi, M.

Nauta, B.-J., Liquidity Risk, Instead of Funding Costs, Leads to a Valuation Adjustment for Derivatives and Other Assets

4 (2015) 1550027

8 (2015) 1550053

1 (2015) 1550004

7 (2015) 1550046

8 (2015) 1550052

1 (2015) 1550007

3 (2015) 1550020

5 (2015) 1550032

3 (2015) 1550020

8 (2015) 1550053

2 (2015) 1550011

4 (2015) 1550025

2 (2015) 1550009

7 (2015) 1550045

7 (2015) 1550047
Neykova, D., see Escobar, M.

Neykova, D., see Escobar, M.

Nguyen, T. A. \& Seifried, F. T., The Multi-Curve Potential Model

Nie, T. \& Rutkowski, M., Fair Bilateral Prices in Bergman's Model with Exogenous Collateralization

Nosrati, A., see Melnikov, A.

Oosterlee, C. W., see Van der Stoep, A. W.

Ortiz-Latorre, S., see Benth, F. E.

Oud, M. A. A. \& Goard, J., Valuation of Options on Oil Futures Under the 3/4 Oil Price Model

Pallavicini, A., see Brigo, D.

Pede, N., see Brigo, D.

Perez-Ostafe, L., see Cordero, F.

Possamaï, D., see Jeanblanc, M.

Protter, P., see Kchia, Y.

$\mathrm{Pu}, \mathrm{J}$, see Guéant, $\mathrm{O}$.

Rebonato, R., ReturnPredicting Factors for US Treasuries: On the Similarity of "Tents" and "Bats"

Réveillac, A., see Jeanblanc, M.

Royer, G., see Guéant, O. Rutkowski, M. \& Tarca, S., Regulatory Capital
5 (2015) 1550033

1 (2015) 1550003

4 (2015) 1550024

5 (2015) 1550033

6 (2015) 1550039

$2(2015) 1550014$

3 (2015) 1550018

5 (2015) 1550030

7 (2015) 1550049

7 (2015) 1550048

7 (2015) 1550047

6 (2015) 1550042

6 (2015) 1550038

8 (2015) 1550050

2 (2015) 1550011

3 (2015) 1550015

5 (2015) 1550029

7 (2015) 1550045

4 (2015) 1550027

3 (2015) 1550019

4 (2015) 1550028

7 (2015) 1550045

3 (2015) 1550019 
Modeling for Credit Risk

Rutkowski, M., see Nie, $\mathrm{T}$.

Sayer, T., see Horsky, R.

Schäfer, R., see Chetalova, D.

Schäfer, R., see Schmitt, T. A.

Schmitt, T. A., Schäfer, R., Dette, H. \& Guhr, T., Quantile Correlations: Uncovering Temporal Dependencies in Financial Time Series

Schmitt, T. A., see Chetalova, D.

Scotti, S., see Bernis, G.

Seifried, F. T., see Desmettre, S.

Seifried, F. T., see Nguyen, T. A.

Serna, G. M., see Jiménez, J. A.

Shen, Y., Anderluh, J. H. M. \& van der Weide, J. A. M., Algorithmic Counterparty Credit Exposure for Multi-Asset Bermudan Options

Siu, T. K., see Elliott, R. J.

Stanley, H. E., see Curme, C.

Subbotin, A., see El Hajjaji, O.

Tarca, S., see Rutkowski, M.
5 (2015) 1550034

7 (2015) 1550048

8 (2015) 1550056

2 (2015) 1550012

7 (2015) 1550044

7 (2015) 1550044

2 (2015) 1550012

1 (2015) 1550002

1 (2015) 1550004

7 (2015) 1550049

8 (2015) 1550051

1 (2015) 1550001

4 (2015) 1550023

7 (2015) 1550043

3 (2015) 1550017

5 (2015) 1550034
Tsuzuki, Y., No-Arbitrage Bounds on Two OneTouch Options

Vaicenavicius, J., see Hambly, B.

Van der Stoep, A. W., Grzelak, L. A. \& Oosterlee, C. W., The TimeDependent FX-SABR Model: Efficient Calibration Based on Effective Parameters

van der Weide, J. A. M., see Shen, Y.

Vargas, V., Dao, T.-L. \& Bouchaud, J.-P., Skew and Implied Leverage Effect: Smile Dynamics Revisited

Vives, J., see Alòs, E.

Vodenska, I., see Curme, C.

Volkmer, H. W., see Feng, R.

Wu, L., CVA and FVA to Derivatives Trades Collateralized by Cash

Yamazaki, K., see Leung, $\mathrm{T}$.

Zagst, R., see Escobar, M.

Zagst, R., see Escobar, M.

Zeng, P., Kwok, Y. K. \& Zheng, W., Fast Hilbert Transform Algorithms for Pricing Discrete Timer Options Under Stochastic Volatility Models

Zhang, H., see Leung, T.

Zheng, W., see Zeng, P.
3 (2015) 1550021

6 (2015) 1550041

6 (2015) 1550042

1 (2015) 1550001

4 (2015) 1550022

6 (2015) 1550036

7 (2015) 1550043

6 (2015) 1550040

5 (2015) 1550035

5 (2015) 1550032

3 (2015) 1550018

5 (2015) 1550030

7 (2015) 1550046

5 (2015) 1550032

7 (2015) 1550046 